Supporting Information for Publication

\title{
Energy integration of a hydrotreatment process for sustainable biojet fuel
} production

Claudia Gutiérrez-Antonio ${ }^{\mathrm{a}^{*}}$, Araceli Guadalupe Romero-Izquierdo ${ }^{\mathrm{b}}$, Fernando Israel GómezCastro $^{\mathrm{b}}$, Salvador Hernández ${ }^{\mathrm{b}}$

a Universidad Autónoma de Querétaro, Facultad de Química, Cerro de las Campanas s/n Col. Las Campanas, Querétaro, Querétaro, 76010, México, *Corresponding author: claudia.gutierrez@uaq.mx

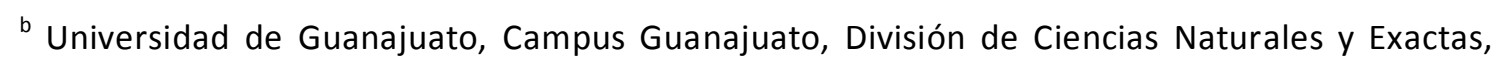
Departamento de Ingeniería Química, Noria Alta s/n, Guanajuato, Guanajuato, 38010, México. 


\section{List of tables}

Table S1. Energy consumption of the equipment required to perform the conditioning of the process streams.

Table S2. Heat exchanger data for the network of scenario 3.

Table S3. Heat exchanger data for the network of scenario 4. 
Table S1. Energy consumption of the equipment required to perform the conditioning of the process streams.

\begin{tabular}{|c|c|c|c|}
\hline \multicolumn{4}{|c|}{ Hydrogen to the reactive section } \\
\hline Compressor 1 & & Heater & \\
\hline Electricity (kW) & 1.78 & Thermal duty (kW) & 28.30 \\
\hline \multicolumn{4}{|c|}{ Jatropha curcas oil to the reactive section } \\
\hline Pump & & Heater & \\
\hline Electricity (kW) & 3.33 & Thermal duty (kW) & 14.02 \\
\hline \multicolumn{4}{|c|}{ Hydrocarbon stream to the separation section } \\
\hline Turbine & & Cooler & \\
\hline Electricity (kW) & 3.366 & Thermal duty (kW) & 28.94 \\
\hline
\end{tabular}


Table S2. Heat exchanger data for the network of scenario 3.

\begin{tabular}{|c|c|c|c|c|c|c|}
\hline \multicolumn{7}{|c|}{ Heat exchanger 1} \\
\hline & \multicolumn{3}{|c|}{ Hot stream: high pressure steam } & \multicolumn{3}{|c|}{ Cold stream: hydrogen } \\
\hline & $\mathrm{T}(\underline{\circ} \mathrm{C})$ & $\mathrm{P}(\mathrm{bar})$ & $\begin{array}{l}\text { Vapor } \\
\text { fraction }\end{array}$ & $\mathrm{T}(\stackrel{\circ}{\mathrm{C}})$ & $\mathrm{P}$ (bar) & Vapor fraction \\
\hline Input & 321.72 & 109.93 & 1 & -192.85 & 80 & 1 \\
\hline Output & 318.06 & 109.93 & 0.15 & -44.5 & 80 & 1 \\
\hline \multicolumn{7}{|c|}{ Heat exchanger 2} \\
\hline & \multicolumn{3}{|c|}{ Hot stream: stream leaving turbine } & \multicolumn{3}{|c|}{ Cold stream: hydrogen } \\
\hline & $\mathrm{T}(\stackrel{\circ}{\mathrm{C}})$ & $\mathrm{P}$ (bar) & $\begin{array}{l}\text { Vapor } \\
\text { fraction }\end{array}$ & $\mathrm{T}(\stackrel{\circ}{\mathrm{C}})$ & $\mathrm{P}$ (bar) & Vapor fraction \\
\hline Input & 408.97 & 1.35 & 1 & -44.5 & 80 & 1 \\
\hline Output & 161.89 & 1.35 & 0.77 & 320 & 80 & 1 \\
\hline \multicolumn{7}{|c|}{ Heat exchanger 3} \\
\hline & \multicolumn{3}{|c|}{$\begin{array}{l}\text { Hot stream: reutilized high pressure } \\
\text { steam }\end{array}$} & \multicolumn{3}{|c|}{ Cold stream: jatropha curcas oil } \\
\hline & $\mathrm{T}(\stackrel{\circ}{\mathrm{C}})$ & $\mathrm{P}$ (bar) & $\begin{array}{l}\text { Vapor } \\
\text { fraction }\end{array}$ & $\mathrm{T}(\stackrel{\circ}{\mathrm{C}})$ & $\mathrm{P}$ (bar) & Vapor fraction \\
\hline Input & 318.06 & 109.93 & 0.15 & 79.95 & 80 & 0 \\
\hline Output & 208.71 & 109.93 & 0 & 190 & 80 & 0 \\
\hline \multicolumn{7}{|c|}{ Heat exchanger 4} \\
\hline & \multicolumn{3}{|c|}{ Hot stream: hydrogen to recirculate } & \multicolumn{3}{|c|}{ Cold stream: jatropha curcas oil } \\
\hline & $\mathrm{T}(\underline{\mathrm{o}} \mathrm{C})$ & $\mathrm{P}$ (bar) & $\begin{array}{l}\text { Vapor } \\
\text { fraction }\end{array}$ & $\mathrm{T}(\stackrel{\circ}{\mathrm{C}})$ & $\mathrm{P}$ (bar) & Vapor fraction \\
\hline Input & 480 & 80 & 1 & 190 & 80 & 0 \\
\hline Output & 298.43 & 80 & 1 & 320 & 80 & 0 \\
\hline \multicolumn{7}{|c|}{ Heat exchanger 5} \\
\hline & \multicolumn{3}{|c|}{ Hot stream: stream leaving turbine } & \multicolumn{3}{|c|}{ Cold stream: cooling water } \\
\hline & $\mathrm{T}(\stackrel{\circ}{\mathrm{C}})$ & $\mathrm{P}($ bar $)$ & $\begin{array}{l}\text { Vapor } \\
\text { fraction }\end{array}$ & $\mathrm{T}(\stackrel{\circ}{\mathrm{C}})$ & $\mathrm{P}(\mathrm{bar})$ & Vapor fraction \\
\hline Input & 161.89 & 1.35 & 0.77 & 25 & 1.01 & 0 \\
\hline Output & 27.22 & 1.35 & 0.38 & 35 & 1.01 & 0 \\
\hline \multicolumn{7}{|c|}{ Heat exchanger 6} \\
\hline & \multicolumn{3}{|c|}{ Hot stream: overheated steam } & \multicolumn{3}{|c|}{ Cold stream: hydrogen to recirculate } \\
\hline & $\mathrm{T}(\stackrel{\circ}{\mathrm{C}})$ & $\mathrm{P}$ (bar) & $\begin{array}{l}\text { Vapor } \\
\text { fraction }\end{array}$ & $\mathrm{T}(\stackrel{\mathrm{o}}{\mathrm{C}})$ & $\mathrm{P}$ (bar) & Vapor fraction \\
\hline Input & 402.97 & 350 & 1 & 298.43 & 80 & 1 \\
\hline Output & 395.27 & 350 & 1 & 320 & 80 & 1 \\
\hline
\end{tabular}


Table S3. Heat exchanger data for the network of scenario 4.

\begin{tabular}{|c|c|c|c|c|c|c|}
\hline \multicolumn{7}{|c|}{ Heat exchanger 1} \\
\hline & \multicolumn{3}{|c|}{ Hot stream: medium pressure steam } & \multicolumn{3}{|c|}{ Cold stream: hydrogen } \\
\hline & $\mathrm{T}(\underline{\mathrm{o}} \mathrm{C})$ & $\mathrm{P}(\mathrm{bar})$ & $\begin{array}{l}\text { Vapor } \\
\text { fraction }\end{array}$ & 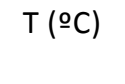 & $\mathrm{P}(\mathrm{bar})$ & Vapor fraction \\
\hline Input & 267.08 & 52.53 & 0.95 & -192.85 & 80 & 1 \\
\hline Output & 267.08 & 52.53 & 0.27 & -44.5 & 80 & 1 \\
\hline \multicolumn{7}{|c|}{ Heat exchanger 2} \\
\hline & \multicolumn{3}{|c|}{ Hot stream: stream leaving turbine } & \multicolumn{3}{|c|}{ Cold stream: hydrogen } \\
\hline & $\mathrm{T}(\stackrel{\circ}{\mathrm{C}})$ & $\mathrm{P}$ (bar) & $\begin{array}{l}\text { Vapor } \\
\text { fraction }\end{array}$ & $\mathrm{T}(\stackrel{\circ}{\mathrm{C}})$ & $P$ (bar) & Vapor fraction \\
\hline Input & 408.97 & 1.35 & 1 & -44.5 & 80 & 1 \\
\hline Output & 161.89 & 1.35 & 0.77 & 320 & 80 & 1 \\
\hline \multicolumn{7}{|c|}{ Heat exchanger 3} \\
\hline & \multicolumn{3}{|c|}{ Hot stream: reutilized pressure steam } & \multicolumn{3}{|c|}{ Cold stream: jatropha curcas oil } \\
\hline & $\mathrm{T}(\underline{\mathrm{o}} \mathrm{C})$ & $\mathrm{P}$ (bar) & $\begin{array}{l}\text { Vapor } \\
\text { fraction }\end{array}$ & $\mathrm{T}(\underline{\mathrm{O}} \mathrm{C})$ & $P($ bar $)$ & Vapor fraction \\
\hline Input & 267.08 & 52.53 & 0.27 & 79.95 & 80 & 0 \\
\hline Output & 204.87 & 52.53 & 0 & 190 & 80 & 0 \\
\hline \multicolumn{7}{|c|}{ Heat exchanger 4} \\
\hline & \multicolumn{3}{|c|}{ Hot stream: hydrogen to recirculate } & \multicolumn{3}{|c|}{ Cold stream: jatropha curcas oil } \\
\hline & $\mathrm{T}(\underline{\mathrm{o}} \mathrm{C})$ & $\mathrm{P}$ (bar) & $\begin{array}{l}\text { Vapor } \\
\text { fraction }\end{array}$ & 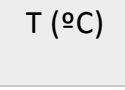 & $P($ bar $)$ & Vapor fraction \\
\hline Input & 480 & 80 & 1 & 190 & 80 & 0 \\
\hline Output & 298.43 & 80 & 1 & 320 & 80 & 0 \\
\hline \multicolumn{7}{|c|}{ Heat exchanger 5} \\
\hline & \multicolumn{3}{|c|}{ Hot stream: stream leaving turbine } & \multicolumn{3}{|c|}{ Cold stream: cooling water } \\
\hline & $\mathrm{T}(\underline{\mathrm{o}} \mathrm{C})$ & $\mathrm{P}$ (bar) & $\begin{array}{l}\text { Vapor } \\
\text { fraction }\end{array}$ & $\mathrm{T}(\stackrel{\mathrm{O}}{\mathrm{C}})$ & $P($ bar $)$ & Vapor fraction \\
\hline Input & 161.89 & 1.35 & 0.77 & 25 & 1.01 & 0 \\
\hline Output & 27.22 & 1.35 & 0.38 & 35 & 1.01 & 0 \\
\hline \multicolumn{7}{|c|}{ Heat exchanger 6} \\
\hline & \multicolumn{3}{|c|}{ Hot stream: overheated steam } & \multicolumn{3}{|c|}{ Cold stream: hydrogen to recirculate } \\
\hline & $\mathrm{T}(\stackrel{\circ}{\mathrm{C}})$ & $\mathrm{P}$ (bar) & $\begin{array}{l}\text { Vapor } \\
\text { fraction }\end{array}$ & $\mathrm{T}(\stackrel{\circ}{ } \mathrm{C})$ & $\mathrm{P}$ (bar) & Vapor fraction \\
\hline Input & 385.21 & 390 & 1 & 298.43 & 80 & 1 \\
\hline Output & 379.83 & 390 & 1 & 320 & 80 & 1 \\
\hline
\end{tabular}

